\title{
Médiévales
}

Langues, Textes, Histoire

62 | printemps 2012

Hagiographie et réforme dans l'Occident latin

\section{De la Rome des papes à la Rome des Romains. À propos de quelques publications récentes sur la Rome médiévale}

\section{Sylvain Parent}

\section{(2) OpenEdition Journals \\ Édition électronique \\ URL : https://journals.openedition.org/medievales/6690 \\ DOI : 10.4000/medievales.6690 \\ ISSN : 1777-5892 \\ Éditeur}

Presses universitaires de Vincennes

\section{Édition imprimée}

Date de publication : 2 juin 2012

Pagination : 175-186

ISBN : 978-2-84292-346-4

ISSN : 0751-2708

Référence électronique

Sylvain Parent, « De la Rome des papes à la Rome des Romains. À propos de quelques publications récentes sur la Rome médiévale », Médiévales [En ligne], 62 I printemps 2012, mis en ligne le 02 juin 2014, consulté le 23 avril 2022. URL : http://journals.openedition.org/medievales/6690 ; DOI : https:// doi.org/10.4000/medievales.6690 
Médiévales 62,printemps 2012, p. 175-186

Sylvain PARENT

\section{DE LA ROME DES PAPES À LA ROME DES ROMAINS. À PROPOS DE QUELQUES PUBLICATIONS RÉCENTES SUR LA ROME MÉDIÉVALE}

À l'exception de rares témoins encore bien conservés, comme la torre delle Milizie ou la torre dei Conti, aux abords des forums impériaux, et de quelques églises qui gardent dans leurs murs et leurs décorations le souvenir de cette période, le Moyen Âge n'imprime que très furtivement sa marque dans la trame urbaine contemporaine de Rome. La quasi-totalité des traces de cet urbanisme médiéval a en effet été emportée par l'«ouragan baroque» (J.-C. Maire Vigueur), qui a modifié en profondeur la topographie de la ville et ferait presque oublier que l'Urbs eut un passé médiéval. Cette absence frappe d'autant plus que de nombreuses communes d'Italie conservent encore un patrimoine médiéval exceptionnel, civil comme ecclésiastique.

Écrire l'histoire de Rome au Moyen Âge représente pour l'historien un redoutable défi, parce que les sources disponibles sont fortement asymétriques, clairsemées, ce qui différencie cette ville de nombre d'autres grandes cités italiennes. Ces carences et ces distorsions ont des explications multiples. Dans certains cas, c'est la destruction pure et simple des archives qui prive les historiens de leur matériau: en la matière, l'exemple le plus révélateur est la disparition des archives de la commune lors du sac de Rome de 1527, à l'exception de quelques épaves documentaires comme les statuts communaux de 1360. Dans d'autre cas, ce peut être l'absence de certains types de sources qui ne semblent pas avoir existé à Rome et que l'on trouve dans d'autres villes - comme les ricordanze ${ }^{1}$. Ce paysage documentaire fragmenté a certainement contribué à

1. C'est l'hypothèse que fait A. Esch. Sur la distorsion produite, voir ID., «Chance et hasard de transmission. Le problème de la représentativité et de la déformation de la transmission historique», dans J.-C. SснмitT et G. OexLe éd., Les Tendances actuelles de l'Histoire du Moyen Âge en France et en Allemagne, Paris, 2000, p. 15-29. 
ancrer durablement l'image négative de Rome, ville sur le déclin et qui serait restée comme à l'écart des mouvements économiques ou artistiques, après les fastes de la période antique. Il explique aussi en grande partie que, pendant très longtemps, comme a pu le suggérer par exemple A. Esch, la Rome des Romains et la commune de Rome aient été largement délaissées par les chercheurs et que l'attention se soit concentrée surtout sur la Rome pontificale et cardinalice, plus facilement saisissable, plus visible ${ }^{2}$. Le livre récent de V. Brancone, $L e$ domus dei cardinali nella Roma del Duecento ${ }^{3}$, sur lequel je reviendrai plus loin, s'inscrit dans cette longue tradition historiographique.

Depuis quelques décennies toutefois, la connaissance de la Rome médiévale a été renouvelée en profondeur. De nouveaux champs d'investigation ont été développés, de nouveaux questionnements ont surgi, permis par le (ré)examen systématique de la documentation disponible et par la réalisation d'opérations archéologiques qui ont fait réapparaître de nombreuses constructions médiévales. On ne peut que se réjouir de la parution récente en français de deux livres qui permettent de prendre la mesure du chemin parcouru. Le premier est la traduction d'un ouvrage collectif dirigé par A. Vauchez, publié en 2001 chez Laterza, puis réédité quelques années plus tard ${ }^{4}$. Il réunit les contributions de treize spécialistes de Rome: G. Barone, M. Boiteux, S. Carocci, P. Delogu,A. Esposito, É. Hubert, J.-C. Maire Vigueur, F. Marazzi, M. Miglio, S. Romano, P. Supino Martini, M. Venditelli et A. Vauchez. Le second, L'Autre Rome. Une histoire des Romains à l'époque communale ${ }^{5}$, est l'œuvre d'un historien spécialiste du monde communal, J.-C. Maire Vigueur, professeur à l'Université de Rome III ${ }^{6}$. Ces deux livres, par la diversité des approches qu'ils proposent, témoignent d'un même souci de faire une histoire de Rome qui déborde le seul cadre pontifical, pour mieux mettre en lumière les aspects trop longtemps laissés dans l'ombre. Leur démarche est néanmoins quelque peu différente: Rome au Moyen Âge, à travers une succession de contributions, fait une mise au point sur de multiples domaines et présente un large panorama actualisé des connaissances sur la longue durée, du $\mathrm{v}^{\mathrm{e}}$ au $\mathrm{Xv}^{\mathrm{e}}$ siècle. L'Autre Rome, véritable essai, se concentre avant tout

2. A. Esch, «Fonti per la storia economica e sociale di Roma nel Rinascimento : un approcio personale», dans A. Esposito et L. PALERMo éd., Economia e società a Roma tra Medioevo e Rinascimento, Rome, 2005, p. 1-31.

3. V. Brancone, Le domus dei cardinali nella Roma del Duecento. Gioielli, mobili, libri, Rome, 2010, 315 p.

4. A. Vauchez dir., Rome au Moyen Âge, Paris, 2010, 520 p.

5. J.-C. Maire Vigueur, L'Autre Rome. Une histoire des Romains à l'époque communale, Paris, 2010, $560 \mathrm{p}$.

6. Il a fait paraître il y a quelques années un autre ouvrage très remarqué sur la militia dans l'Italie communale: Cavaliers et citoyens. Guerre, conflits et société dans l'Italie communale XIIXIII $s$., Paris, 2003. 
sur cette Rome méconnue et longtemps délaissée, voire dénigrée, celle de la commune, entre le XII et le XIV ${ }^{\mathrm{e}}$ siècle, et porte une ambition forte: sortir l'Urbs de l'étrangeté dans laquelle elle a été tenue durant des décennies, procéder à sa «réhabilitation» historique, et rappeler que dans le «domaine de l'économie et de la société, [Rome a] une histoire aussi digne d'intérêt que celle de toute autre grande ville de l'Italie communale» (p. 118). Disons-le d'emblée : la force de ce livre érudit, précis et très clair, tient aussi à son style, qui met le lecteur dans la posture du voyageur médiéval découvrant progressivement la ville, ses monuments antiques, ses églises, ses dizaines de tours, ses quartiers et son effervescence. De ce point de vue, l'ouvrage dirigé par A. Vauchez ne suscite pas un semblable plaisir de lecture - sans que cela ne remette nullement en cause la qualité de son contenu -, peut-être tout simplement parce qu'il est composé à plusieurs voix et qu'il est donc moins homogène (il faut ajouter à cela que la qualité de la traduction des articles italiens est assez inégale et dessert parfois le texte).

Ces publications sont à ce point foisonnantes qu'il n'est évidemment pas envisageable, dans le cadre restreint de ce point de vue, d'emprunter chacune des pistes qu'elles explorent. Aussi ai-je choisi, dans les pages qui suivent, de sélectionner quelques-uns des thèmes qui y sont développés.

\section{Entre «normalité» et «anomalie»: une histoire politique de la Rome médiévale}

Rome au Moyen Âge et L'Autre Rome dressent une vaste fresque de l'histoire politique de la Rome médiévale depuis le très haut Moyen Âge (voir les contributions de P. Delogu, «Le passage de l'Antiquité au Moyen Âge» et de F. Marazzi, «Aristocratie et société, $\mathrm{VI}^{\mathrm{e}}-\mathrm{XI}^{\mathrm{e}}$ siècles » dans Rome au Moyen $\hat{A} g e)$. Cependant, c'est incontestablement pour la Rome communale (11431398) que les éléments les plus stimulants sont apportés, sous la plume de J.-C. Maire Vigueur en particulier, qui est aussi l'auteur du chapitre consacré à cette période dans l'ouvrage dirigé par A. Vauchez. La réflexion qu'il mène sur les évolutions du régime politique vise à rappeler à quel point Rome fut une «commune normale» (p. 204), tout en insistant cependant sur les éléments qui peuvent, à certains moments de son histoire, la distinguer des autres communes. L'émergence du groupe des barons et leur forte présence sur la scène romaine, en particulier entre les années 1250 et 1350, a constitué l'une des principales « anomalies ».

J.-C. Maire Vigueur revient notamment sur la «naissance théâtrale» de la commune, en 1143, «un des événements les plus décisifs de l'histoire de Rome» (p. 305): le peuple prend d'assaut le Capitole, procède à la restauration du Sénat 
(cette renovatio senatus évoquée par Otton de Freising), libérant ainsi la ville de la tutelle pontificale. Cependant, dénonçant la lecture trop simpliste qui, pendant longtemps, a été faite de cet événement, l'auteur balaie deux idées reçues: il nuance tout d'abord fortement la fulgurance de l'événement, son caractère «révolutionnaire », rappelant que le passage à une complète indépendance visà-vis du pouvoir pontifical s'est fait progressivement, dans les décennies qui l'ont précédé - des sénateurs sont par exemple déjà attestés seize ans avant cette date. Ensuite, c'est peut-être surtout le caractère populaire de ce qui fut un temps considéré comme une «révolution bourgeoise et démocratique» (p. 209) qui doit être reconsidéré: loin de signifier l'irruption du peuple sur la scène politique romaine, la prise de pouvoir de 1143 marque au contraire l'avènement de l'ordo equestris - terme employé par Otton de Freising -, c'est-à-dire de la noblesse urbaine, cette militia dont l'auteur a analysé toute la complexité dans son ouvrage précédent ${ }^{7}$. Elle occupe à partir de ce moment non seulement les fonctions sénatoriales mais aussi les charges publiques, et ce jusqu'au milieu du XIII siècle: sur ce point précis, Rome se démarque des autres communes où l'on voit se développer la magistrature du consulat, propre à cette première période du mouvement communal italien. La commune qui émerge en 1143 est donc bien une «commune de cavaliers citoyens» (p. 305). Elle se dote en revanche d'un parlement et de conseils, ce qui, de ce point de vue, ne la distingue pas fondamentalement des autres cités. Durant la période où la puissance des barons est à son paroxysme, entre le milieu du XIII ${ }^{e}$ et le milieu du XIV siècle, Rome voit se produire une douzaine de changements de régimes: c'est à la faveur de l'un d'eux qu'émerge Cola di Rienzo, éphémère seigneur de la ville entre mai et décembre 1347. Les pages consacrées à la dernière phase de l'existence de la commune de Rome, après la chute de Cola di Rienzo, insistent sur le rôle de plus en plus important que joue le popolo dans la vie politique romaine, notamment dans les conflits de factions qui déchirent les familles baronniales les plus puissantes: durant ces années d'instabilité, la ville vit «une des expériences les plus singulières de toute l'histoire communale italienne» (p. 354) avec la Felice Società dei Balestrieri e dei Pavesati, société armée et populaire qui constitue une forme originale de gouvernement populaire. L'aventure communale s'achève en juillet 1398, lorsque la commune remet son pouvoir aux mains de la papauté.

En matière politique, la commune de Rome, tout au long de son existence, revendiqua un héritage: celui de l'Antiquité. Dans un ultime chapitre très dense et stimulant consacré à ses multiples usages (chapitre VIII, «Du bon (et du moins bon) usage de l'Antiquité »), J.-C. Maire Vigueur propose une réflexion sur les modèles politiques et juridiques qui ont façonné l'expérience communale

7. J.-C. Maire Vigueur, Cavaliers et citoyens... 
romaine. Une double référence est mobilisée. La commune affirme en premier lieu sa volonté de continuité avec l'Empire romain, dont elle se considère comme l'héritière directe. Elle entend ainsi, de fait, participer pleinement à l'élection des empereurs - une prétention que ces derniers, à l'image de Frédéric II, n'acceptèrent évidemment pas. Le point culminant de cette revendication, sa plus radicale explicitation, est constitué par le «grand show» (p. 475) orchestré par Cola di Rienzo au Latran en 1346: devant la foule assemblée, il lit et commente la lex regia, gravée sur une plaque de bronze conservée dans la basilique. Cette loi avait été édictée sous Vespasien - elle fut incorporée ensuite dans le Code Justinien - et rappelait que les empereurs recevaient leur imperium du Sénat, octroyé au nom du peuple romain. Cette vigueur de la référence impériale ne semble cependant pas exclure, loin de là, la survie d'un autre modèle fort, celui de la Rome républicaine: J.-C. Maire Vigueur en décèle les manifestations dans deux moments clés de l'histoire de la commune, d'abord dans les années 11401150, au moment de la naissance de la commune, qui aurait cherché à imiter en quelque sorte les institutions de la République, puis plus tard, peu de temps avant l'arrivée au pouvoir de Cola di Rienzo, dont Pétrarque fait un troisième Brutus qui «a libéré Rome de la tyrannie des barons» (p. 484) et qui prend le titre très républicain de tribun. Ces pages passionnantes s'achèvent sur le récit de l'échec cinglant du projet - anachronique - porté par Cola di Rienzo et qui transparaît dans les cérémonies grandioses qu'il organise à l'été 1347: celui d'une Italie unifiée par un pouvoir impérial fort.

\section{Une histoire sociale et économique de la Rome des Romains}

Comme en témoignent les riches contributions de S. Carocci, É. Hubert, J.-C. Maire Vigueur et M. Venditelli, c'est sans aucun doute du point de vue de l'étude des structures sociales et économiques que nos connaissances ont le plus progressé.

Sur le plan social, ces deux ouvrages offrent une réflexion sur la construction des identités, à tous les niveaux de la société. Pendant longtemps, l'attention s'est concentrée de façon privilégiée sur le groupe restreint des familles baronniales (Orsini, Capocci, Normanni, Conti, Annibaldi, etc.) au sommet de la hiérarchie citadine, un groupe dont S. Carocci s'est fait l'historien ${ }^{8}$ et qui, selon lui, a développé une forte "conscience de classe». Composées d'une dizaine de lignages qui ont émergé de l'aristocratie romaine à l'issue d'un processus de sélection interne, ces familles aux «revenus dignes du CAC 40» (J.-C. Maire Vigueur) ont joué un grand rôle dans la vie romaine des XIII et $\mathrm{XIV}^{\mathrm{e}}$ siècles, au

8. S. CARocCi, Baroni di Roma. Dominazioni signorili e lignaggi aristocratici nel duecento e nel primo trecento, Rome, 1993. 
plan politique comme économique. Si elles constituent une «anomalie» aux yeux de J.-C. Maire Vigueur, c'est en partie parce que l'emprise qu'elles imposent au cours de ces décennies sur les hommes et sur les terres qu'elles possèdent autour de Rome va à l'encontre du mouvement observé à l'échelle de l'Occident, qui voit les hommes s'émanciper des tutelles seigneuriales. Les barons ne représentent pas pour autant toute la noblesse, loin de là, et J.-C. Maire Vigueur, dans L'Autre Rome, redonne toute sa place à cette noblesse citadine de cavaliers citoyens, composée de 200 à 300 familles, qui fut jusqu'à l'émergence des barons la seule élite citadine et qui conserva une forte continuité tout au long de la période (chapitre IV, «La noblesse citadine: métamorphoses et recomposition d'une classe sociale»). Dans la synthèse dirigée par A. Vauchez, où la part belle est donnée aux franges supérieures de la société, on peut en revanche s'étonner de l'absence quasi complète d'un acteur majeur: le peuple. L'un des nombreux mérites du livre de J.-C. Maire Vigueur est justement de lui rendre la place qui lui revient (chapitre III, «Le peuple de Rome»), en partie grâce à l'exploitation des registres notariés, dont une centaine est conservée pour la deuxième moitié du XIV ${ }^{\mathrm{e}}$ siècle. Cela donne lieu à de très belles pages sur le monde des métiers (bouchers, poissonniers, artisans, aubergistes, meuniers et autres potiers), sur l'organisation des corporations - semblable à celle de bien d'autres villes de l'Italie communale -, ou sur les fêtes qui animent régulièrement la cité et dont certaines, à l'image de la fête du Testaccio, deviennent au cours du XIV siècle de grandes fêtes identitaires du popolo. Les contributions d'É. Hubert dans Rome au Moyen Âge («L'organisation territoriale et l'urbanisation») et les réflexions de J. C. Maire Vigueur dans L'Autre Rome rappellent que ces identités se construisent dans un cadre qui demeure encore fortement revendiqué dans la Rome d'aujourd'hui : celui des quartiers, les rioni, vecteurs de cohésion (les membres d'une même profession se regroupent dans un même quartier) et cadres privilégiés d'organisation de la vie politique.

Ces deux livres permettent ainsi de faire le point sur l'activité et les structures économiques de la ville pour mieux en faire ressortir toute la complexité et le dynamisme. Une fois encore, les principales difficultés en la matière tiennent à l'état contrasté de la documentation, et ce sont ici avant tout les registres notariés et les documents conservés dans les fonds des églises qui s'avèrent les plus utiles. Parmi ces champs d'investigation qui ont été très féconds au cours des dernières décennies figure notamment celui des relations que la commune de Rome, véritable «mastodonte territorial» (J.-C. Maire Vigueur) de $1700 \mathrm{~km}^{2}$, entretient avec sa campagne occupée par plusieurs centaines de grands domaines, les casali (on en compte 400 dans un rayon de 20 à 30 kilomètres autour de Rome), où l'on pratique surtout l'élevage et la culture céréalière et qui se perpétuent jusqu'au début du $\mathrm{xx}^{\mathrm{e}}$ siècle. Les travaux de 
S. Carocci, M. Venditelli ${ }^{9}$ ou encore J.-C. Maire Vigueur ont bien montré que ce processus d' «incasalamento » a été dans une très large mesure le fait de la noblesse citadine, et en son sein notamment des familles qui se livrent aux affaires ou aux activités commerciales. C'est à M. Venditelli que l'on doit, en particulier, d'avoir redécouvert le rôle joué par ce groupe des mercatores romani, dont il situe l'âge d'or dans les années 1150-1230; ses membres, qui sont parfaitement intégrés dans les circuits commerciaux internationaux, comme le montre la présence des Romains aux foires de Champagne, a pleinement contribué au développement économique de la ville et certains d'entre eux ont entretenu des relations privilégiées avec la cour pontificale. S. Carocci, M. Venditelli et J.-C. Maire Vigueur restituent ensuite au fil des pages les rythmes complexes de l'économie romaine entre le milieu du XIII ${ }^{\mathrm{e}}$ siècle et le début du $\mathrm{Xv}^{\mathrm{e}}$ siècle, période durant laquelle la ville est largement sous l'emprise des barons, mais qui voit également la papauté s'éloigner: toute la difficulté est bien sûr de mesurer l'impact de ces phénomènes à partir des sources disponibles. Il faut attendre la fin du XIV siècle pour voir se mettre en place de nouvelles dynamiques qui font changer de rythme l'économie romaine.

Ces recherches, on l'aura compris, en faisant apparaître sous un nouveau jour des pans entiers de la société et de l'économie urbaines, contribuent à libérer Rome de l'image dont elle était prisonnière depuis l'époque moderne: celle d'une ville peu dynamique économiquement, voire paresseuse. Une image d'ailleurs encore très largement exploitée, comme le rappelle J.-C. Maire Vigueur, par les leaders de certains partis politiques populistes contemporains - la Ligue du Nord par exemple - qui dénoncent une Rome «vivant aux crochets de la seule Italie qui travaille et produise des richesses, celle des villes du Nord» (p. 117).

\section{La Rome des cardinaux}

La multiplication des travaux sur l'histoire économique et sociale n'a évidemment pas épuisé l'intérêt porté à la Rome ecclésiastique : intérêt d'une part pour le patrimoine religieux de la ville, ses églises et monastères en particulier, leurs évolutions architecturales et artistiques. Sur ce point, les pages écrites par J.-C. Maire Vigueur dans L'Autre Rome (chapitre VII, «Les couleurs de la ville») et par S. Romano, grande spécialiste de la peinture romaine, dans Rome au Moyen Âge («L'art du Moyen Âge romain»), apportent des éléments très solides, qui contribuent à redonner à Rome la place qui lui revient en matière de dynamisme artistique et revalorisent son rôle par rapport à celui de Florence. J.-C. Maire Vigueur situe cet «âge d'or» de la production artistique romaine entre

9. Voir en particulier S. CARocci et M. Venditelli, L'Origine della Campagna Romana : casali, castelli e villaggi nel XII e XIII secolo, Rome, 2004. 
la fin du XIII et le tout début du XIV ${ }^{\mathrm{e}}$ siècle, moment où de nombreux chantiers sont engagés, au Vatican, dans les grandes basiliques et plus largement dans de nombreuses églises de la ville - en partie grâce à l'argent des cardinaux. Parmi les peintres, sculpteurs et mosaïstes de renom qui furent actifs dans ces décennies, on trouve ainsi Arnolfo di Cambio, Giotto, Jacopo Torriti, Pietro Cavallini ou Filippo Rusuti. L'intérêt s'est d'autre part porté sur l'évolution des structures ecclésiastiques et sur ses composantes : la contribution de G. Barone dans Rome au Moyen Âge («clercs, moines et frères »), montre bien que papes et cardinaux ont fait pendant longtemps l'objet de toutes les attentions, laissant quelque peu dans l'ombre l'étude du clergé romain alors que, selon elle, ce sont justement ces religieux (chanoines, recteurs ou prêtres) qui «ont modelé la religiosité des habitants de l'Urbs, bien plus que les papes et les cardinaux» (p. 281). Cette asymétrie s'explique, une fois de plus, en partie par l'état des sources disponibles, qui rendent les enquêtes sur les franges inférieures de la hiérarchie ecclésiastique difficiles et souvent partielles. Il n'est donc guère étonnant que les cardinaux aient eu la part belle et que ce milieu soit sans aucun doute celui que nous connaissons désormais le mieux, en particulier grâce aux travaux d'A. Paravicini Bagliani ${ }^{10}$ ou encore ceux de S. Carocci, auteur d'un essai sur les pratiques népotistes de la Rome pontificale et cardinalice ${ }^{11}$. Ces pratiques profitent surtout aux familles d'origine romaine: entre 1188 et 1216, une vingtaine de cardinaux est ainsi issue de la noblesse romaine.

Le livre de V. Brancone s'inscrit donc dans cette longue tradition d'étude de la société cardinalice et vient compléter les travaux disponibles. Après un premier opus publié en 2009 sur les inventaires après décès des cardinaux du

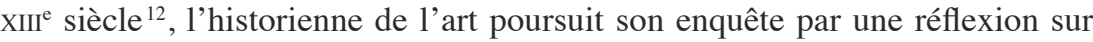
les domus des cardinaux dans un livre paru chez Viella et qui constitue le dixneuvième volume d'une collection de grande qualité dirigée par A. Paravicini Bagliani ( «La corte dei papi») ${ }^{13}$. L'auteur concentre de nouveau ses analyses sur le XIII ${ }^{\mathrm{e}}$ siècle, au cours duquel le cardinalat connaît un profond renouvellement, et revendique clairement deux objectifs : écrire une synthèse sur ces demeures cardinalices, pour lesquelles nous disposons déjà d'une bibliographie bien fournie ; ensuite, inscrire l'histoire des trésors cardinalices dans une histoire plus large de la thésaurisation privée au Moyen Âge, un thème qui connaît lui aussi un regain

10. Au sein d'une bibliographie immense, citons Cardinali di Curia e 'familiae' cardinalizie dal 1227 al 1254, Padoue, 1972 et I testamenti dei cardinali del Duecento, Rome, 1980.

11. S. CARocci, Il nepotismo nel medioevo. Papi, cardinali e famiglie Nobili, Rome, 1999.

12. V. BRANCONE, Il tesoro dei cardinali del Duecento. Inventari di libri e beni mobili, Florence, 2009.

13. Sur les premiers titres de cette collection, voir V. THEIS, «Images de l'institution pontificale. Revue critique de la collection 'La corte dei papi' (Viella)», Médiévales, 45 (2003), p. 159-172. 
d'intérêt depuis quelques années ${ }^{14}$. L'entreprise est d'autant plus périlleuse que la quasi-totalité de ces palais cardinalices a été détruite et qu'il n'en existe, pour le $\mathrm{XIII}^{\mathrm{e}}$ siècle, aucune source descriptive. Ainsi, à part quelques témoignages architecturaux ou picturaux résiduels, c'est en partie dans la documentation écrite, quand celle-ci ne fait pas défaut, que l'histoire de ces domus doit être cherchée - ce qui soulève, une fois encore, bien des difficultés. L'ouvrage se divise en six chapitres: dans les cinq premiers, V. Brancone convie le lecteur à un parcours qui le mène de l'extérieur vers l'intérieur, des complexes palatiaux vers les chambres des résidences, au plus près de ces trésors cardinalices. Le sixième et dernier chapitre consiste en un très riche glossaire des termes liés au trésor et à la thésaurisation (p. 209-268). Les données proprement architecturales étant très minces, ce livre est avant tout une enquête sur le vocabulaire.

Le premier chapitre tente de cerner les réalités qui se cachent derrière le terme palatium, dont la définition pose beaucoup de difficultés. Dans la Rome du XIII ${ }^{\mathrm{e}}$ siècle, le palatium semble être la demeure noble par excellence. Comme l'ont bien montré par ailleurs les travaux de S. Carocci, l'habitude prise par nombre de prélats de s'installer dans des résidences souvent très luxueuses est à mettre en grande partie sur le compte des liens que ces prélats entretenaient avec les familles baronniales. Les domus cardinalices n'ayant pas survécu aux remodelages multiples que la ville a connus depuis l'époque moderne, l'auteur doit donc essayer de trouver ailleurs les modèles qui permettent d'expliquer l'évolution architecturale de ces palatia cardinalices: elle invoque pour cela la nécessité d'inscrire ces demeures romaines dans un contexte italien et européen plus large, prenant appui en particulier sur l'observation des résidences épiscopales ou archiépiscopales. Elle justifie un tel déplacement de la focale par le fait que beaucoup de cardinaux du XIII ${ }^{e}$ siècle avaient été à la tête d'évêchés ou d'archevêchés: une fois nommés et installés à Rome, ils n'auraient donc en quelque sorte fait que reproduire des schémas architecturaux auxquels ils étaient habitués. Le modèle formel de palais qui se met en place dans ce cadre est alors centré sur un noyau aula-camera-capella, ces palais se présentant notamment sous la forme de maisons-tours hautes et étroites, composées de pièces qui occupent l'ensemble des niveaux. C'est en partie dans les demeures papales de Rome et du Patrimoine de Saint-Pierre qu'elle tente de trouver des modèles de référence (chap. 2), en cherchant à en comprendre l'agencement, l'organisation et les liens avec les autres parties de la demeure: la tour d'Innocent III au Vatican, ou encore celles construites dans les palais de Viterbe, Pérouse ou Orvieto en constituent des exemples. Les usages de chacune des pièces de ces tours palatiales soulèvent bien des débats parmi les spécialistes, et notamment

14. Cf. par exemple L. Burkart, P. Cordez, P. A. Mariaux et Y. Potin éd., Le Trésor au Moyen Âge. Discours, pratiques et objets, Florence, 2010. 
les liens qu'elles entretiennent entre elles - de ce point de vue, l'auteur n'est pas en mesure d'apporter beaucoup d'éléments nouveaux. Ces apories la conduisent à un retour sur les modèles résidentiels romains, en s'appuyant sur les travaux de R. Krautheimer et de S. Carocci : le premier avait proposé quelques modèles résidentiels de base, soulignant par exemple la constance du réemploi des édifices ou encore l'exigence de décor et de lustre des familles de l'aristocratie, ou insistant sur le fait que les nombreuses tours qui sont construites durant l'époque communale ne sont jamais isolées mais au contraire toujours intégrées dans des complexes architectoniques plus vastes composés de plusieurs maisons; la dichotomie entre barons et noblesse a entraîné des formes spécifiques et dissociées d'insediamento, les palais des barons, en général érigés à l'intérieur d'ensemble fortifiés plus vastes, dépassant en luxe et en ostentation toutes les autres résidences. Mais là encore, la plupart des palais cardinalices provenant de ces familles baronniales n'ont pratiquement pas survécu et on en sait très peu de choses. Le chapitre suivant (3) se concentre alors sur trois complexes cardinalices. Le premier est celui des Santi Quattro Coronati, bâti sur des édifices remontant à l'époque carolingienne et adossé à la basilique. Le cardinal Stefano Conti y résida au cours des deux premières décennies du XIII ${ }^{\mathrm{e}}$ siècle et y fit construire la Torre Maggiore: le rez-de-chaussée accueillait la chapelle, le premier étage était réservé à la grande aula et l'étage supérieur consistait en une terrasse qui fut par la suite recouverte d'une toiture. Sur le flanc oriental de la torre Maggiore une tour plus étroite a été édifiée - très certainement par le cardinal Ottaviano degli Ubaldi - et certains ont pu faire l'hypothèse que cette seconde tour était destinée aux appartements du cardinal (cette hypothèse est avancée sur la base d'une comparaison avec les appartements pontificaux qui se trouvaient près de la tour d'Innocent III au Vatican). Le second édifice pris en compte est celui de San Clemente, non loin du Colisée. Durant la quasi-totalité de la première moitié du XIII ${ }^{\mathrm{e}}$ siècle, l'administrateur de cette église et de son couvent fut le cardinal Raniero Capocci : aucun document n'a été conservé, mais l'on sait simplement qu'une tour d'assez grande dimension y fut édifiée entre 1216 et 1246, dont il subsiste quatre étages et des restes de fresques, analysées par S. Romano. Enfin, un complexe similaire a été construit auprès de l'église des Santi Giovanni e Paolo, où une tour semblable à celle de San Clemente fut érigée.

Au terme de cette succession de chapitres où l'auteur part en quête de modèles architecturaux pertinents et fiables, le livre pénètre plus avant dans ces palais et se concentre dès lors sur les espaces d'habitation de ces demeures pour essayer d'en déterminer la fonction et surtout d'identifier les lieux de conservations des trésors accumulés par les cardinaux (chapitre 4). Une fois de plus, les difficultés semblent être quasi insurmontables. L'enquête se fait lexicale et tourne ainsi autour de la définition de notions telles que thesaurus, archivum, sagrestia, vestiarum, ainsi que sur l'identification de pièces évoquées dans les 
chapitres précédents, comme la capella, l'aula, le studium ou la camera. Dans le cas des chapelles, par exemple, les allusions conservées dans les documents permettent d'affirmer que les chapelles cardinalices ne peuvent pas être confondues avec les églises limitrophes et extérieures aux palais mais seraient bien des édifices qui font corps avec le palais : l'inventaire du cardinal Luca Fieschi, qui résida dans le palais attenant à Santa Maria Maggiore, donne une idée de l'opulence des biens que pouvaient contenir ces chapelles. Mais encore plus que la chapelle, c'est peut-être la camera qui semble être le lieu par excellence de conservation du trésor. Si les incertitudes demeurent si grandes au terme de ces pages, c'est en grande partie parce que ces espaces ont certainement une vocation multifonctionnelle que le vocabulaire ne permet pas de saisir complètement. Enfin, ultime étape, V. Brancone achève son parcours par une analyse des meubles et des objets que contiennent ces trésors (chapitre 5). Elle souligne d'emblée une sorte de paradoxe: la notion même de «trésor» pour définir les collections d'objets précieux des cardinaux du XIII ${ }^{\mathrm{e}}$ siècle est selon elle impropre, en ceci qu'elle ne reflète pas la réalité terminologique transmise par les sources qui décrivent la composition de ces trésors - en particulier les inventaires ; pourtant, ces collections cardinalices finissent par incarner l'image traditionnelle du trésor, à cause des modalités d'accumulation et d'usage ou encore par la recherche délibérée des pièces choisies. Rejoignant ici un terrain connu qui avait fait l'objet de son livre précédent, elle montre que ces inventaires des biens mobiliers des cardinaux du XIII ${ }^{\mathrm{e}}$ siècle fonctionnent en quelque sorte comme une «photographie instantanée». Une «photographie» dont il faut se méfier puisque l'inventaire n'est pas nécessairement exhaustif - certaines parties du trésor pouvant être cachées, conservées dans d'autres demeures ou destinées à un autre siège. L'autre point faible de ces inventaires est qu'ils ne permettent pas de déduire les lieux où sont conservés les objets, en fonction de leur nature par exemple. Autrement dit, le trésor du cardinal du XIII ${ }^{\mathrm{e}}$ siècle est un trésor dispersé : entre des lieux divers au sein même du complexe cardinalice d'une part, mais également entre ses différentes résidences, entre les sacristies des institutions ecclésiastiques administrées par le cardinal ou encore auprès des marchands ou des personnes de confiance. Pour donner au lecteur une idée de l'opulence des biens possédés par ces cardinaux, V. Brancone décrit par le détail les biens du cardinal Luca Fieschi: des livres, des vêtements et tissus, des bijoux et des pierres précieuses, des objets servant à la table du cardinal par exemple 41 poti dévolus à l'eau et au vin -, des ornements liturgiques, etc. L'objet le plus précieux de cette étonnante accumulation est une mitre estimée à 2000 florins. Élargissant l'horizon, la réflexion de l'auteur se pose pour terminer sur le problème de la constitution de ces collections d'objets et sur les intentions ou choix qui y président: si les cardinaux ne sont pas selon elle à proprement parler des collectionneurs, au sens où nous l'entendons aujourd'hui, leurs trésors 
semblent néanmoins déjà appartenir à ce qu'elle appelle une «communauté de goût» (p. 178).

Toutes ces publications, portées par des ambitions différentes, attestent de la diversité des recherches sur Rome en ce début du XXI ${ }^{\mathrm{e}}$ siècle, des plus traditionnelles aux plus novatrices. De cet ensemble émerge incontestablement un grand livre d'histoire, L'Autre Rome : tout en faisant le point sur les travaux de plusieurs générations d'historiens de la Rome médiévale, il ouvre de nombreuses pistes nouvelles et modifie en profondeur la vision que l'on pouvait se faire d'une ville qui, il n'est désormais plus permis d'en douter, est inscrite de plain-pied dans l'histoire du mouvement communal italien.

Sylvain PARent - École normale supérieure de Lyon, CIHAM-UMR 5648, sylvain.parent@ens-lyon.fr. 
Médiévales 62,printemps 2012, p. 187-194

\section{NOTES DE LECTURE}

Hélène Couderc-Barraud, La Violence, l'ordre et la paix. Résoudre les conflits en Gascogne du XI $e^{e}$ au début du XIII siècle, Toulouse, Presses Universitaires du Mirail, $2008,377 \mathrm{p}$.

Le choix de l'illustration de couverture, une miniature du Beatus de Saint-Sever représentant l'un des quatre cavaliers de l'Apocalypse, démontre le degré de raffinement atteint par les moines de l'abbaye de Saint-Sever en Gascogne au $\mathrm{XI}^{\mathrm{e}}$ siècle. C'est en effet dans cette région qu'Hélène Couderc-Barraud a décidé de comprendre comment se déroulait la résolution des conflits entre le XI $\mathrm{I}^{\mathrm{e}}$ siècle et le XIII' siècle. L'ouvrage, issu d'une thèse de doctorat soutenue en 2005, est au cœur du renouvellement historiographique du Moyen Âge central à l'œuvre ces deux dernières décennies, qui interroge les thèses de l'anarchie féodale et des violences perpétuelles de l'aristocratie.

L'analyse des conflits permet de mettre en évidence l'ensemble du système social de la Gascogne. Pour cela, l'auteur a traqué toutes les mentions et formes de conflits dans un large corpus documentaire composé, en premier lieu, de cartulaires et de chartriers essentiellement monastiques, mais aussi épiscopaux et laïques. Ces derniers sont un atout permettant d'élargir le regard issu des religieux dont les écrits sont majoritaires. Pour compléter, des règlements normatifs de villes et de villages, tant du Béarn que de la Bigorre, sont mis à contribution. La méthodologie adoptée a permis d'effectuer une étude précise du vocabulaire des conflits dans l'ensemble des actes. Ainsi, le mot violence dans les processus de résolution est-il examiné pour comprendre tous les sens qu'il implique à cette époque. La violence fait d'ailleurs l'objet d'un chapitre entier (p. 195242). De même, pour mesurer l'émiettement des pouvoirs, sont analysées avec précision les désignations des membres de l'aristocratie intervenant dans les règlements, ainsi que les mots des procédures permettant de mesurer une évolution géographique dans les modes de résolutions.

La démonstration commence par l'examen de l'autorité des puissances publiques en confrontant les textes normatifs à la réalité, c'est-à-dire si les princes réussissent à régler les conflits ou à les faire régler par leurs représentants - les comtes de Bigorre, par exemple, ne pouvant pas juger directement. Le conflit entre le comte de Bigorre et Sanche Garsie, un noble puissant de la région qui conteste la puissance du comte, met en évidence les mécanismes de la résolution des conflits à l'intérieur d'une société féodale vers 1125. Il est ainsi fait appel au roi d'Aragon, seigneur des deux parties, pour conclure les différends. Cela démontre la place de la hiérarchie féodale et les liens d'homme à homme en Gascogne. En Béarn, les vicomtes semblent plus à même d'établir leur autorité, et donc leur justice, sur la population et les seigneurs. D'une manière générale, les puissances laïques du territoire réussissent à imposer leur justice, d'une façon plus 
ou moins complète selon les cas. Les plaintes montent dans la hiérarchie seigneuriale en fonction de la difficulté à les résoudre ; l'auteur parle d'une « gradation dans le recours au tiers » (p. 89). L'émergence progressive de communautés organisées dans les villages et dans les villes a pour conséquence une évolution notable: les probi homines de ces lieux sont appelés plus souvent à témoigner, voire à juger, démontrant la place plus grande des entités villageoises et urbaines à partir de la seconde moitié du XII ${ }^{\mathrm{e}}$ siècle. La procédure est également l'objet d'une gradation puisque les causes arrivent progressivement au plaid.

Concernant les justices ecclésiastiques, H. C.-B. s'intéresse à l'effectivité des droits octroyés par l'immunité aux monastères, comme la délégation de la justice publique. Finalement, cela dépend des personnes à juger: l'effectivité des décisions sera plus grande sur les religieux que sur les puissants laïcs. Compte tenu des bornes chronologiques de l'ouvrage, celui-ci se situe en plein dans les débuts de la réforme dite grégorienne qui intensifie les interventions du pape et de ses prélats lors des conflits au sein de l'Église gasconne, avec parfois de fortes résistances à une intervention vécue comme une ingérence.

Une fois les justices étudiées, c'est au tour des justiciables, «des paysans aux bourgeois ». Il est bien sûr très difficile d'approcher par les sources les paysans. Les habitats groupés qui se constituent sont quelquefois l'objet de conflits entre seigneurs désireux de les posséder. Toutefois, les seigneurs laïques ont bien compris qu'ils devaient traiter les villes et les bourgs comme des alliés, notamment lors de conflits armés. Enfin, la façon dont les conflits étaient résolus est analysée en détail, la signification de la violence recevant une attention particulière. L'auteure montre notamment avec justesse que la violence est un élément constitutif de la résolution des conflits. En dernier recours, les litiges, notamment ceux concernant des héritages, peuvent mener à des morts. D'ailleurs, les questions économiques, l'enjeu de la constitution d'un patrimoine et des moyens de l'accroître et de le conserver, auraient pu faire l'objet d'un petit chapitre. En effet, pratiquement tous les conflits évoqués portent sur des biens ou sur le contrôle des hommes rapportant des redevances. Quant aux procédures de résolution, elles sont étudiées en profondeur: du plaid aux différents éléments acceptés pour preuve, aux différences entre justices ecclésiastiques et laïques ainsi que les écarts géographiques, ou encore l'évolution de la place de l'écrit.

L'ensemble du conflit et de sa résolution est scruté, les hommes appelés à témoigner sont dûment identifiés, les liens familiaux retracés permettant des analyses et des conclusions solides; tous les acteurs de la régulation sociale sont ainsi examinés. De même, les différences géographiques dans la résolution des conflits sont mises en évidence ainsi que l'autorité des puissances publiques selon les régions. L'étude rend donc compte avec finesse de l'importance de la résolution des conflits et de la complexité de la société médiévale entre le $\mathrm{XI}^{\mathrm{e}}$ et le XIII ${ }^{\mathrm{e}}$ siècle. 
Scott G. Bruce, Silence and Sign Language in Medieval Monasticism. The Cluniac Tradition (ca 900-1200), Cambridge, Cambridge University Press, 2009 [2007], 209 p. (Cambridge Studies in Medieval Life and Thought, Fourth Series, 68)

Les anges vivent dans le silence et la psalmodie; les hommes dans la confusion des langues. Cette idée-force, si chère aux ascètes chrétiens des premiers siècles qui ont abandonné le bruit des villes à la recherche des milieux peu peuplés, est au centre de l'argumentation du beau livre de Scott G. Bruce. Entièrement basé sur une documentation écrite - avant tout normative, et particulièrement coutumière -, le livre est bien cadré dans le champ analytique de l'idéation, à savoir le rôle de l'analogie «moine-ange» dans la normalisation de la communication quotidienne des moines. Si nous pouvons le croire présent depuis les débuts du monachisme, l'idéal d'abandon de la parole n'était pas homogène, comme l'auteur le démontre bien. Pendant des siècles, ce renoncement a été au centre des polémiques monastiques, devenant largement accepté au XII ${ }^{\mathrm{e}}$ siècle seulement, après le tournant fondamental orchestré à Cluny.

En opposition à la tradition classique qui associe le beau parler à l'activité politique citoyenne, le monachisme de l'Antiquité tardive valorise le silence en tant qu'exercice spirituel ascétique et discipline sociale. Scott G. Bruce soutient que cette ligne de conduite a eu une double fonction : éviter de laisser le moine être séduit par les aménités d'une rhétorique peccamineuse, ainsi que favoriser l'humilité et l'obéissance, aspects primordiaux de la société monastique. Cela ne signifie pas que les religieux de l'Antiquité tardive ont abandonné la parole, mais plutôt que l'idéal de silence jouait un rôle de grande importance dans l'organisation sociale par le biais du contrôle des formes de communication au sein de la communauté. Le désir du silence à l'époque carolingienne a surtout encouragé l'état révérenciel des moines et la distinction entre la quiétude du cloître et le bruit du monde. Finalement, le $x^{\mathrm{e}}$ siècle, au-delà de la réaffirmation des topiques de l'humilité et de l'obéissance, attribue à la métaphore moine-ange un aspect apocalyptique de première importance: les moines de Cluny essayent de reproduire sur terre le silence éternel des êtres supérieurs qui accompagnera la résurrection du Christ à la fin du monde. Ce changement de perspective a trouvé des résistances et a pris à Cluny un aspect particulier chez les ascètes de l'époque.

Parmi les trois qualités angéliques fondamentales (pureté sexuelle, psalmodie et silence), les clunisiens sont connus par leur effort d'émulation des éléments sonores, qui les amène à concevoir le silence comme une vertu en soi-même. Il s'agit d'une «nouvelle idéologie de l'ascétisme chrétien qui associe la glorification du silence à l'idéal d'une vie angélique accomplie dans un corps mortel ». C'est bien là que se trouve le fondement de leur prestige auprès des laïcs et d'autres membres de l'Église : l'efficacité de leurs oraisons est assurée par la diffusion de l'acceptation de l'idéologie à la base de leur fonction dans la société. Le silence est ainsi un créateur de distinction sociale, fait fort intéressant sousjacent au plan argumentatif du livre, mais non exploité par l'auteur dans sa dimension d'élément structurant de l'institution ecclésiale.

D'une écriture très agréable rendant sa lecture accessible à tous les publics, le livre est divisé en cinq chapitres. Le premier («Uttering No Human Sound») aborde les réussites et les limites de la discipline du silence chez les moines de Cluny vis-à-vis de la logique du rapport moine-ange. En partant de l'acte de donation de Guillaume III, duc d'Aquitaine, des textes d'Odon, deuxième abbé de Cluny (927-942), ainsi que de la vita Odonis rédigée 
par Jean de Salerne, Scott G. Bruce met au premier plan l'emploi du vocable coelestis comme qualificatif de la vie menée par les moines de Cluny. Selon l'auteur, l'exhortation contre le discours mondain et en faveur de son contrôle est une des principales marques de l'abbatiat d'Odon. De même, l'approche, établie par Jean de Salerne, entre les clunisiens et la tradition du silence sacré, diffusé par la Bible et par les premiers ermites chrétiens, permet à l'auteur de visiter la topique de l'isolement monastique. Cette perspective n'empêche pas son contraire. En effet, la place privilégiée donnée au silence à Cluny, selon Jean de Salerne, attirait la critique des contemporains qui la comprenaient comme une création nouvelle. Il faudra attendre deux siècles pour que cette méfiance se transforme en éloge, après l'établissement d'un langage de signes bien cadré.

Le chapitre suivant («The Training of the Hand») présente le lexique des signes pratiqué à Cluny au $\mathrm{XI}^{\mathrm{e}}$ siècle afin de démontrer son aspect linguistique. Le langage de signes créé à Cluny vise les activités quotidiennes élémentaires comme l'alimentation et la correction des mœurs, notamment ceux des novices. Basé sur un répertoire de noms sans rapport avec des structures complexes, il s'agit d'un langage volontairement simple, et non d'un système trop proche du langage parlé qui pourrait faciliter une communication de type mondain et, par conséquent, mettre en cause le silence lui-même. Ensuite («A Silent Commerce of Signs »), nous passons à une enquête sur les fonctions et l'application des signes dans les coutumes de Bernard de Cluny et d'Ulrich de Zell (fin Xi ${ }^{e}$ siècle). Documentation à l'appui, l'auteur soutient que l'apparition et l'importance du langage des signes à Cluny au XI ${ }^{\mathrm{e}}$ siècle sont une solution efficace dans un milieu plurilinguistique où les accents et les connaissances du latin poseraient des difficultés à la communication quotidienne. Le cas d'Orderic Vital est cité afin de démontrer comment les différents accents du latin médiéval sont créateurs de difficultés de communication et des sentiments d'identité.

Dans l'étape suivante («Transmission and Adaptation»), l'argumentation trace l'expansion et les modifications des signes monastiques au-delà de la Bourgogne. L'auteur vise à établir l'influence de Cluny dans le contenu des trois autres codes de gestes produits avant le XII ${ }^{\mathrm{e}}$ siècle, à Fleury, à Canterbury et à Hirsau. Les manuscrits rédigés dans ces maisons ne présentent pas des principes de langage au-delà de la structure sémiotique clunisienne et, par conséquent, ne dépassent pas les fonctions de la communication silencieuse dans l'abbaye. Depuis le début du XI ${ }^{\mathrm{e}}$ siècle, Fleury possède un lexique propre, de base clunisienne, mais adapté à la coutume locale. Canterbury, selon l'auteur, aurait organisé son système autour de l'an mil, après la visite d'un moine de Fleury, maison réformée par Odon de Cluny vers 930. Là aussi, le langage a maintenu la simplicité tout en étant enrichi par le vocabulaire local. Finalement, S.G. Bruce soutient que le fort aspect liturgique du lexique composé dans les années 1080 à Hirsau ne s'oppose pas au caractère didactique de la liste de Cluny. Bien au contraire. Les deux catalogues peuvent être compris comme étant complémentaires, si l'on pense que les moines de la Forêt Noire ont, peut-être, tout simplement mis par écrit ce qui était pratiqué, mais non rédigé, à Cluny - à cause de la fonction didactique-moralisante du langage clunisien .

Le dernier chapitre («Continuity and Criticism») offre une analyse de l'expansion et de l'adaptation des signes au XII siècle. L'auteur aborde l'idéal du silence et la pratique des signes chez les cisterciens (continuité de la valorisation du silence et des gestes), les chanoines réguliers (valorisation des gestes et critique du silence) et la Grande Chartreuse 